\title{
Retrait-gonflement des argiles, proposition de méthodologie
}

\section{Shrinkage and swelling of clayey soils, proposal for methodology}

\author{
G. PHILIPPONNAT \\ Ingénieur ETP - Directeur technique de SOPENA SA
}

Rev. Franç. Géotech. n 57 , pp. 5-22 (octobre 1991)

\section{Résumé}

La sécheresse de l'été 1990 a conduit en France à de très nombreux sinistres affectant les constructions légères et montrant l'importance que revêt l'étude des sols dits gonflants.

Des procédures d'essais sont d'abord présentées afin d'appréhender d'une façon simple mais quantitative les phénomènes de retrait et gonflement. Ensuite une approche de la prévision des amplitudes de mouvements de sol dus à ces phénomènes est tentée.

Enfin des méthodes de calcul sont proposées par la prise en compte des effets parasites sur les fondations profondes et semi-profondes. En annexe figure une méthode générale de calcul aux états limites des fondations semi-profondes (puits coulés à pleine fouille).

\begin{abstract}
The drought which occured during the 1990 summer created many disasters affecting light buildings and showed the necessity of studying the expansive soils.

Procedures for tests are described with the object of doing a simply but quantitative estimation of the shrinkage and swelling of soils. Then an approach of predictions of the shrinkage or swelling amplitude is made. Last, computation methods are propounded to take in account the secondary forces applied to deep and semi-deep foundations. A general method for limites states calculation of semi-deep foundations is also given.
\end{abstract}




\section{INTRODUCTION}

Après 1976, les annèes sèches successives de 1989 et 1990 sont lourdement ressenties par les assureurs.

Les sinistres dus aux phénomènes de retrait et gonflement notamment sur les pavillons et constructions légères sont de plus en plus nombreux ou tout du moins mieux identifiés par les experts.

Nous avions attiré l'attention, il y a déjà un certain temps (PHILIPPONNAT, 1978), sur l'importance de ces problèmes et proposé un moyen simple pour identifier les sols gonflants, alors que dans les décennies précédentes, on avait tendance à penser qu'il s'agissait de problèmes frappant les pays à climat semiaride ou aride.

De son côté la Grande-Bretagne, également affectée, menait des recherches très instructives (DRISCOLL, 1983).

Parallèlement l'ISSMFE a créé un comité technique sur les sols expansifs (groupe TC6).

En dehors de l'organisation de congrès internationaux périodiques sur le sujet, le comité TC6 a publié un projet de norme pour a l'évaluation de la pression de gonflement des sols expansifs en laboratoire (ISSMFE, 1990).

Il est encore également intéressant de citer un ouvrage majeur sur le sujet (F.H. CHEN, 1988). L'auteur s'est consacré depuis de très nombreuses années à l'étude des argiles gonflantes. Cependant, la lecture de cet ouvrage montre que, bien qu'il soit signalé que le retrait est un phénomène indissociable du gonflement et que celui-ci peut provoquer des désordres aussi importants, le seul sujet faisant l'objet d'un examen approfondi est celui du gonflement.

Sous le climat métropolitain et notamment dans la moitié nord, les argiles situées à faible profondeur sont souvent déconsolidées, humidifiées et ont épuisé leur potentiel de gonflement à l'état naturel. Mais elles sont dans un état éloigné de leur limite de retrait et peuvent se rétracter si leur teneur en eau diminue de façon notable.

Par contre en profondeur, ces mêmes argiles sont souvent surconsolidées et donc susceptibles de gonfler si elles sont déchargées ou si elles voient leur teneur en eau augmenter (argile verte, argile plastique).

On voit donc que des sinistres peuvent survenir tant par retrait que par gonflement. Personnellement, nous avons constaté de très nombreux désordres dus au retrait mais également un nombre non négligeable dû au gonflement.

Les points suivants vont être abordés :

1. proposition d'une méthodologie d'essais de laboratoire en vue d'une normalisation. Ces essais permettent de quantifier les deux phénomènes de retrait et de gonflement;

2. présentation d'une méthode d'interprétation ayant pour but la prévision de l'amplitude des phénomènes tout au moins dans certains cas simples;
3. méthodes de calcul pour la prise en compte des effets de retrait-gonflement dans le dimensionnement des fondations.

\section{ESSAIS DE LABORATOIRE}

\subsection{Identification des argiles gonflantes}

Plusieurs types d'approche sont décrits par différents auteurs. Cependant les spécialistes s'accordent sur la fiabilité très limitée des méthodes indirectes, par exemple à partir des caractéristiques d'identification, et sur l'intérêt de la mesure directe à l'aide de l'odomètre.

Comme nous l'avons montré (G. PHILIPPONNAT, 1985), le paramètre le plus significatif permettant d'identifier les sols expansifs est la pente de la courbe de déchargement de l'œilomètre $(\mathrm{Cg})$.

La pression de gonflement $\left(\sigma_{\mathrm{g}}\right)$ n'est pas une caractéristique intrinsèque du sol mais est fonction de son état d'humidité au moment de l'essai. La mesure de la pression de gonflement n'a de sens que si le sol testé est à l'état naturel dans un état de succion élevé et ne peut en aucun cas avertir des risques liés au retrait.

\subsection{Mesure du potentiel de gonflement. Détermination de la pression de gonflement}

L'essai proposé correspond pratiquement au projet de normalisation du comité TC6 de la société internationale de mécanique des sols (voir annexe 1).

Il consiste à placer plusieurs éprouvettes, découpées dans le même échantillon, dans des bâtis œdométriques sous des pressions normales faibles et croissantes, puis à alimenter chaque éprouvette en eau et à mesurer le gonflement linéaire après stabilisation.

L'objectif de cet essai n'est pas seulement de mesurer la pression de gonflement $\sigma_{g}$ mais consiste à déterminer la loi entre lamplitude de gonflement $\delta \mathrm{H} / \mathrm{H}$ et la pression normale appliquée $\sigma_{\mathrm{v}} \leq \sigma_{\mathrm{g}}$.

La figure 1 montre le gonflement linéaire relatif $\delta \mathrm{H} / \mathrm{H}$ (\%) qui se produit lorsque le sol supportant une contrainte totale $\sigma_{\mathrm{v}}$ inférieure à $\sigma_{\mathrm{g}}$ est soumis à une alimentation en eau libre, c'est-à-dire lorsque la succion devient nulle.

Il est admis par le projet de norme précité que la relation entre $\delta \mathrm{h} / \mathrm{h}$ et $\log \left(\sigma_{\mathrm{v}}\right)$ est linéaire. Dans les assez nombreux essais que nous avons réalisés, les résultats peuvent être souvent assimilés à ce cas (fig. 1), il arrive également que la courbe $\delta \mathrm{h} / \mathrm{h}=\mathrm{f}(\log \sigma)$ ait l'allure indiquée sur la figure 2 (courbe en trait plein).

$\mathrm{Si}$ on admet une relation linéaire, l'intersection de cette droite avec l'axe des $\sigma_{\mathrm{v}}$ fournit la valeur de la pression de gonflement. Il est cependant préférable de poursuivre les essais jusqu'à une pression telle qu'un tassement soit observé à la mise en eau ; la pression de gonflement est alors obtenue par l'intersection de la courbe $\delta \mathrm{h} / \mathrm{h}=\mathrm{f}(\log \sigma)$ avec l'axe des abscisses que cette courbe soit une droite ou non (fig. 2). 


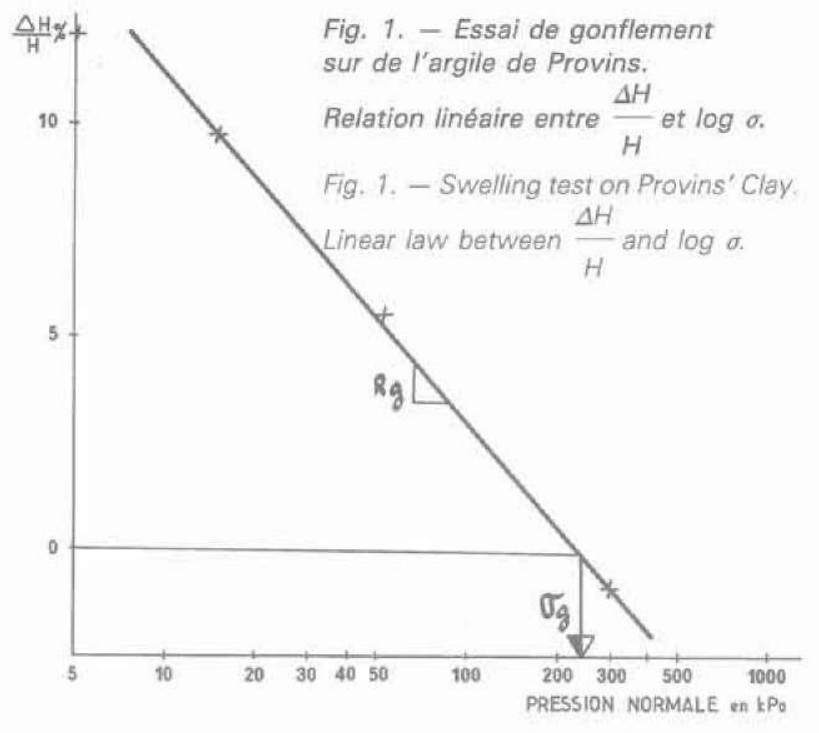

PRESSION DE GONFLEMENI $240 \mathrm{kPo}$ RAPPORT DE GONFLEMENT $: 8.10^{-2}$

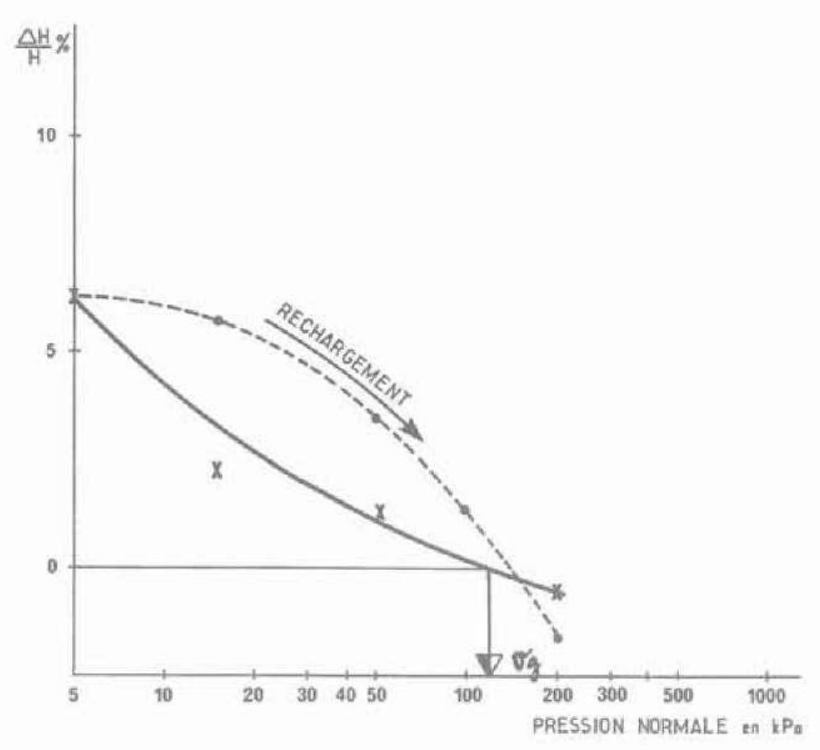

PRESSION DE GONFLEMENT: $110 \mathrm{kP}$ Fig. 2. - Essai de gonflement. Loi hyperbolique entre $\frac{\Delta H}{H}$
et $\log \sigma$. Courbe de rechargement.

Fig. 2. - Swelling test. Hyperbolic law between $\frac{\Delta H}{H}$

\subsubsection{Définition du rapport de gonflement}

Toujours avec l'hypothèse d'une relation linéaire, la loi de gonflement dans une plage de pression verticale $\sigma_{\mathrm{v}}$ allant de $1 \mathrm{kPa}$ à $\sigma_{\mathrm{g}}$ s'écrit :

$$
\frac{\delta H}{H}=-R G \cdot \log \left(\sigma_{g} / \sigma_{v}\right)
$$

avec : RG $=$ rapport de gonflement.

\subsubsection{Expression mathématique du gonflement non linéaire}

Dans le cas plus général ou la relation n'est pas linéaire, on peut exprimer le gonflement par la formule suivante (courbe hyperbolique) :

$$
\frac{\delta \mathrm{h}}{\mathrm{h}}=\frac{\mathrm{a}}{\log \sigma_{\mathrm{v}}-\mathrm{l}_{0}}+\mathrm{dh}_{0}
$$

Les coefficients $\mathrm{a}, \mathrm{l}_{0}$ et $\mathrm{dh}_{0}$ étant calés sur trois points de la courbe expérimentale.

Nota :

- Il a été adopté un symbole différent (RG) du coefficient de gonflement afin de ne pas entraîner de confusion avec le coefficient $\mathrm{c}_{\mathrm{g}}$ mesuré à l'œdomètre (pente de la courbe de retour).

- On pourrait également s'orienter vers un essai réalisé sur une éprouvette unique, par exemple méthode A de la norme ASTM D 4546 (1986) ou essais proposés par G. DIDIER (G. DIDIER et al., 1987).

L'essai ASTM consiste à provoquer le gonflement de l'éprouvette sous la surcharge la plus faible $(5 \mathrm{kPa}$ par exemple) puis à recharger l'éprouvette par paliers comme pour un œedomètre classique jusqu'à ce que le volume initial de l'échantillon soit atteint à nouveau, ce qui correspond à la pression de gonflement. Cependant les essais comparatifs auxquels nous nous sommes livrés semblent montrer que si les pressions de gonflement obtenues par les deux méthodes sont assez voisines, par contre l'allure des courbes $\delta \mathrm{h} / \mathrm{h}$ $=f(\log \sigma)$ est très différente (fig. 2). Ce dernier essai ne permet pas de faire une prévision des amplitudes de gonflement pour des études de cas.

- Certains auteurs proposent de reconsolider l'éprouvette sous le poids des terres $\sigma_{\circ}$ avant de commencer l'essai.

Pour les problèmes de fondations, la contrainte dans le massif sous la semelle est différente de $\sigma_{0}$ lorsqu'un gonflement consécutif à une arrivée d'eau se produit. Cette opération qui alourdit la procédure de l'essai paraît superfétatoire pour les études courantes.

\subsection{Essai de retrait}

\subsubsection{Description de l'essai de retrait au mercure} Dans le même esprit, l'essai de retrait n'a pas seulement pour objet de déterminer la limite de retrait mais aussi de quantifier les variations dimensionnelles du sol entre l'état de teneur en eau naturelle initial et un état de dessiccation donné.

Une série d'essais a été réalisée de la façon suivante : - mesure du poids et du volume initial d'un petit échantillon intact ou reconstitué de sol de forme cylindrique (mesure du volume par immersion dans un bain de mercure) ;

- on laisse l'échantillon se dessécher à l'air et on mesure à différents intervalles de temps son poids et son volume par le même procédé ;

- dessiccation totale de l'éprouvette à l'étude et mesure de son poids et de son volume final.

Les éprouvettes avaient un diamètre initial de $36 \mathrm{~mm}$ et une hauteur de $20 \mathrm{~mm}$.

Le matériel nécessaire à cet essai est très simple et identique à celui utilisé pour la mesure de la limite de retrait classique (R. PELTIER, 1959).

A partir de cet essai, il est possible de tracer, entre autres, deux courbes qui ont l'allure de la figure 3 . 

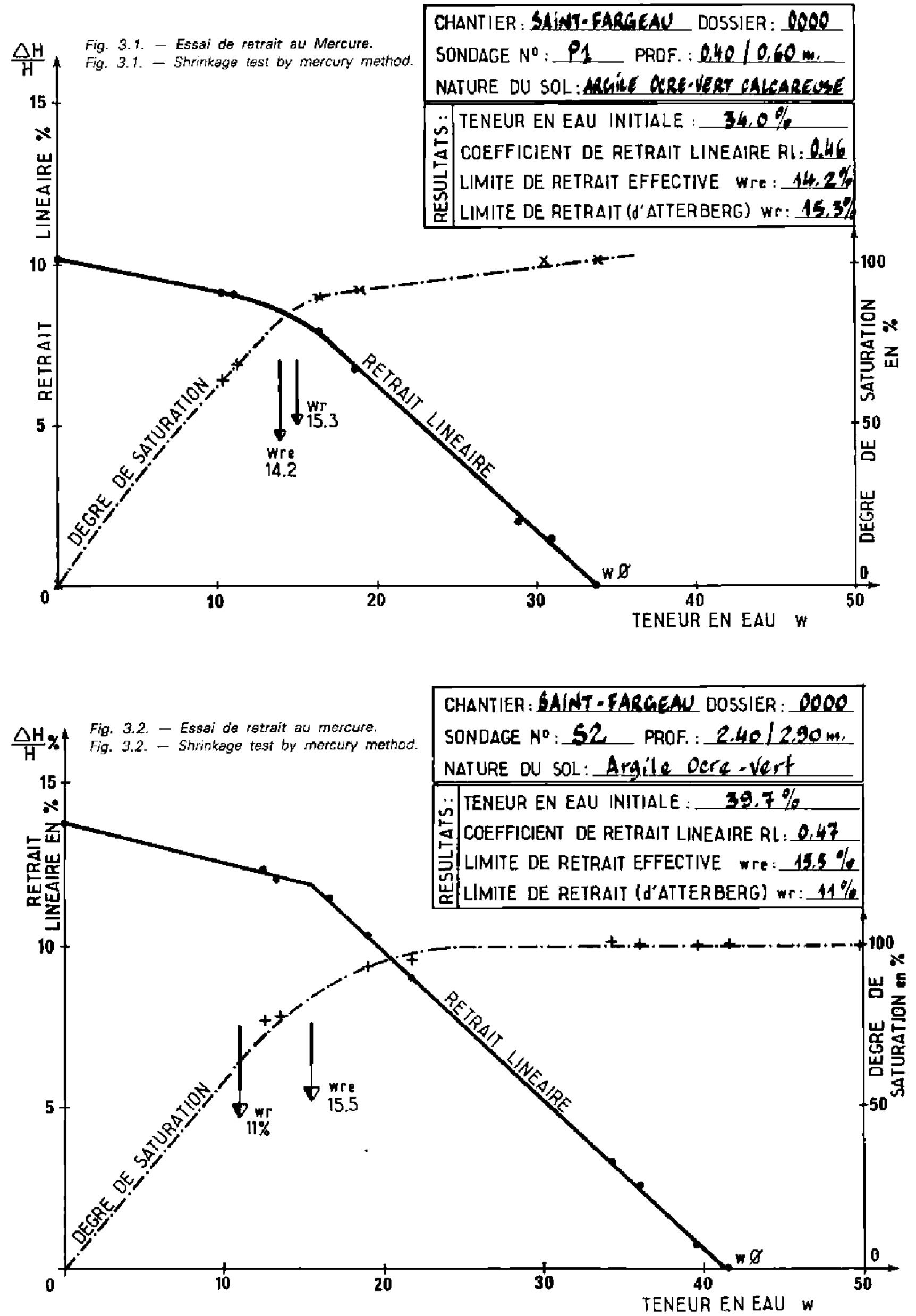


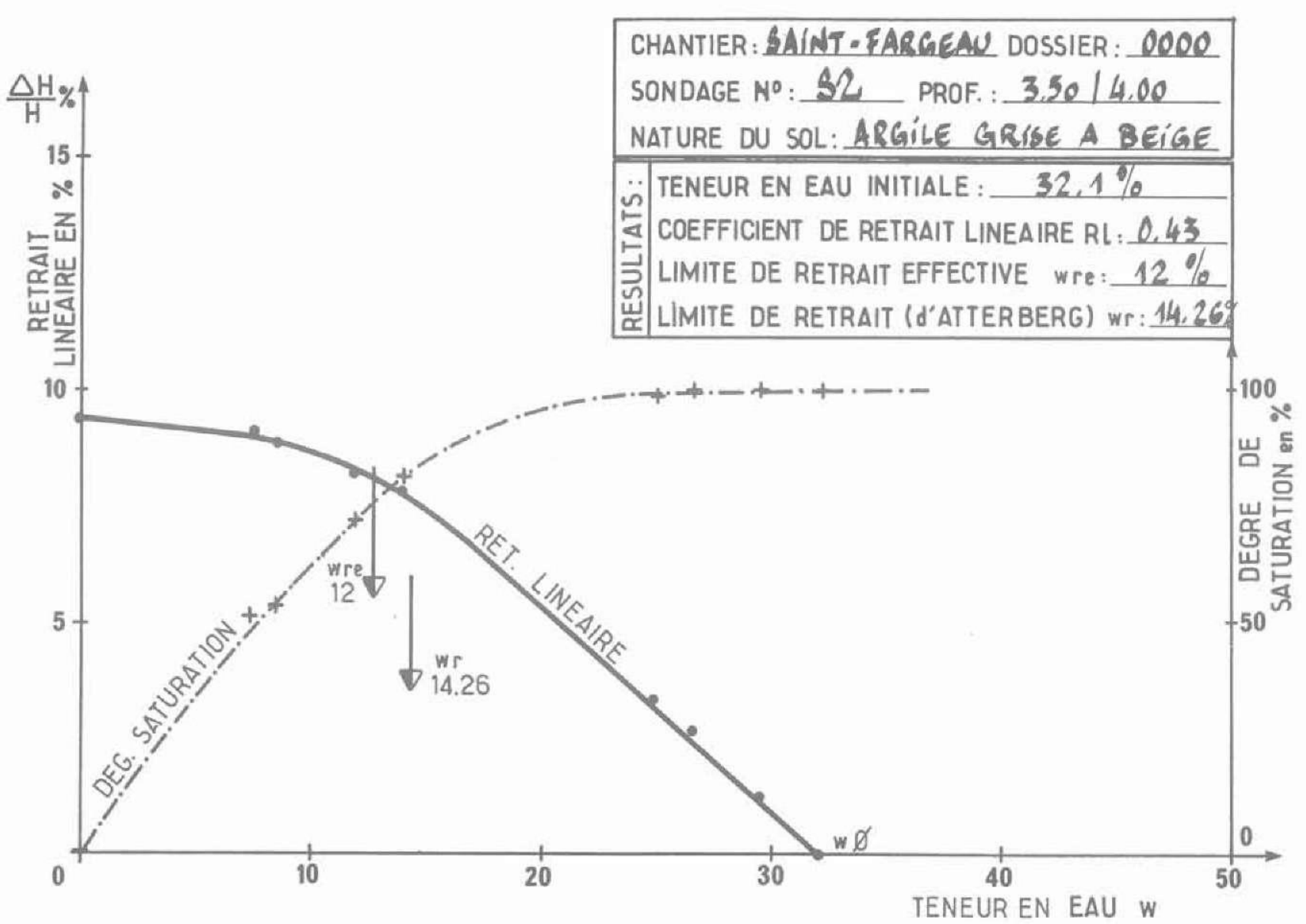

Fig. 3.3. - Essai de retrait au mercure.

Fig. 3.3. - Shrinkage test by mercury method.

Où :

$w_{0}$ est la teneur en eau initiale de l'échantillon ;

$\delta \mathrm{H} / \mathrm{H}_{\mathrm{o}}$ est le retrait linéaire tel que :

$$
\left(1-\frac{\delta \mathrm{H}}{\mathrm{H}_{\mathrm{o}}}\right)=\left(1-\frac{\delta \mathrm{V}}{\mathrm{v}_{\mathrm{o}}}\right)^{1 / 3}
$$

$\mathrm{H}$ et $\mathrm{V}=$ hauteur et volume de l'échantillon pour une teneur en eau $w$;

$\mathrm{H}_{0}$ et $\mathrm{v}_{\mathrm{o}}=$ hauteur et volume initiaux de l'échantillon.

Sur cette figure sont représentés :

- la courbe du retrait linéaire mesuré qui traduit les variations de $\delta \mathrm{H} / \mathrm{H}_{\mathrm{o}}$ en fonction de $w$;

- la courbe du degré de saturation calculé qui traduit les variations du degré de saturation en fonction de $w$;

- la valeur $\omega_{r}$ de la limite de retrait mesurée selon le mode opératoire des limites d'Atterberg ;

- la valeur de la limite de retrait déduite du présent essai et définie ci-après par «limite de retrait effective " $w_{\text {re. }}$.

Les essais réalisés montrent que la courbe de retrait linéaire comporte deux branches quasi-rectilignes. La branche fortement inclinée correspond au retrait du sol avant qu'une désaturation importante ne se produise et la courbe peu inclinée au retrait résiduel du sol éloigné de la saturation. La pente de la première branche est appelée coefficient de retrait linéaire: $\mathrm{R}_{1}$.

Nous proposons de considérer comme limite de retrait effective $w_{\text {re }}$, la teneur en eau correspondant à l'intersection de ces deux branches.

Bien entendu, on peut définir de la même façon un retrait volumique unitaire liant $\delta \mathrm{v} / \mathrm{v}$ à $\delta \mathrm{w}$ mais ce coefficient apparaît moins utile pratiquement.

\subsubsection{Exemples de résultats}

Les figures 3.1 . à 3.3 . présentent les résultats de trois essais réalisés sur une argile verte du Sannoisien dans un site où une construction est affectée par de graves désordres suite à la sécheresse de 1990.

Les trois essais correspondent à des prélèvements effectués à des profondeurs différentes et à deux emplacements différents.

On constate :

- la concordance des résultats des trois essais ;

- l'importance des variations dimensionnelles dues au retrait, c'est ainsi qu'une perte de teneur en eau de 5 points sur une épaisseur de $2 \mathrm{~m}$ conduit en gros 
à un tassement de 4 à $5 \mathrm{~cm}$, toutes choses égales par ailleurs.

Les figures 4.1. à 4.3. fournissent les résultats de trois essais réalisés dans un limon argileux puis une argile limoneuse situés au contact des limons des plateaux et de l'argile à meulière (Marne-la-Vallée).

Là encore, aux dispersions des mesures près, on obtient une assez bonne concordance entre la limite de retrait mesurée selon le mode opératoire d'Atterberg et la limite de retrait effective.

Les essais réalisés à 1,$0 ; 1,5 \mathrm{~m}$ et 2,$0 ; 2,5 \mathrm{~m}$ montrent des sols peu sensibles au retrait, ce qui n'est plus le cas pour l'argile limoneuse plus profonde.

\subsubsection{Vérification de la validité de la formule (2)}

Plusieurs essais de retrait ont été réalisés avec un mode opératoire similaire mais en mesurant séparément les variations de diamètre et de hauteur au pied à coulisse.

Ceci a permis de mesurer directement $\delta \mathrm{H} / \mathrm{H}$ et de le comparer à $\delta \mathrm{V} / \mathrm{V}$.

Les points expérimentaux présentent une certaine dispersion car la mesure au pied à coulisse n'est pas aisée à faire sans abîmer l'échantillon.
La figure 5 présente des résultats obtenus sur huit essais provenant de deux sites différents.

On observe un assez bon agrément avec la relation théorique de la formule 2 , celle-ci semblant avoir une tendance à fournir une valeur légèrement surestimée de $\delta \mathrm{H} / \mathrm{H}$. Compte tenu des objectifs recherchés, cette formule peu être considérée comme valable.

\subsubsection{Propositions pour un essai normalisé}

L'essai au mercure tel qu'il a été pratiqué, présente des inconvénients, ceci malgré les bons résultats obtenus :

- la manipulation du mercure est à éviter pour des raisons d'hygiène et de sécurité ;

- les manipulations répétées des éprouvettes pour chaque opération de mesure de volume par immersion dans le mercure et de pesée sont délicates et peuvent conduire à une détérioration de ces dernières.

Il est préférable de s'orienter vers une mesure directe de $\delta \mathrm{H} / \mathrm{H}$ avec un dispositif tel que celui représenté sur la figure 6 .

Ce système permettrait d'éviter toute manipulation directe de l'éprouvette qui resterait à demeure sur son support.

Un prototype est en cours d'étude.

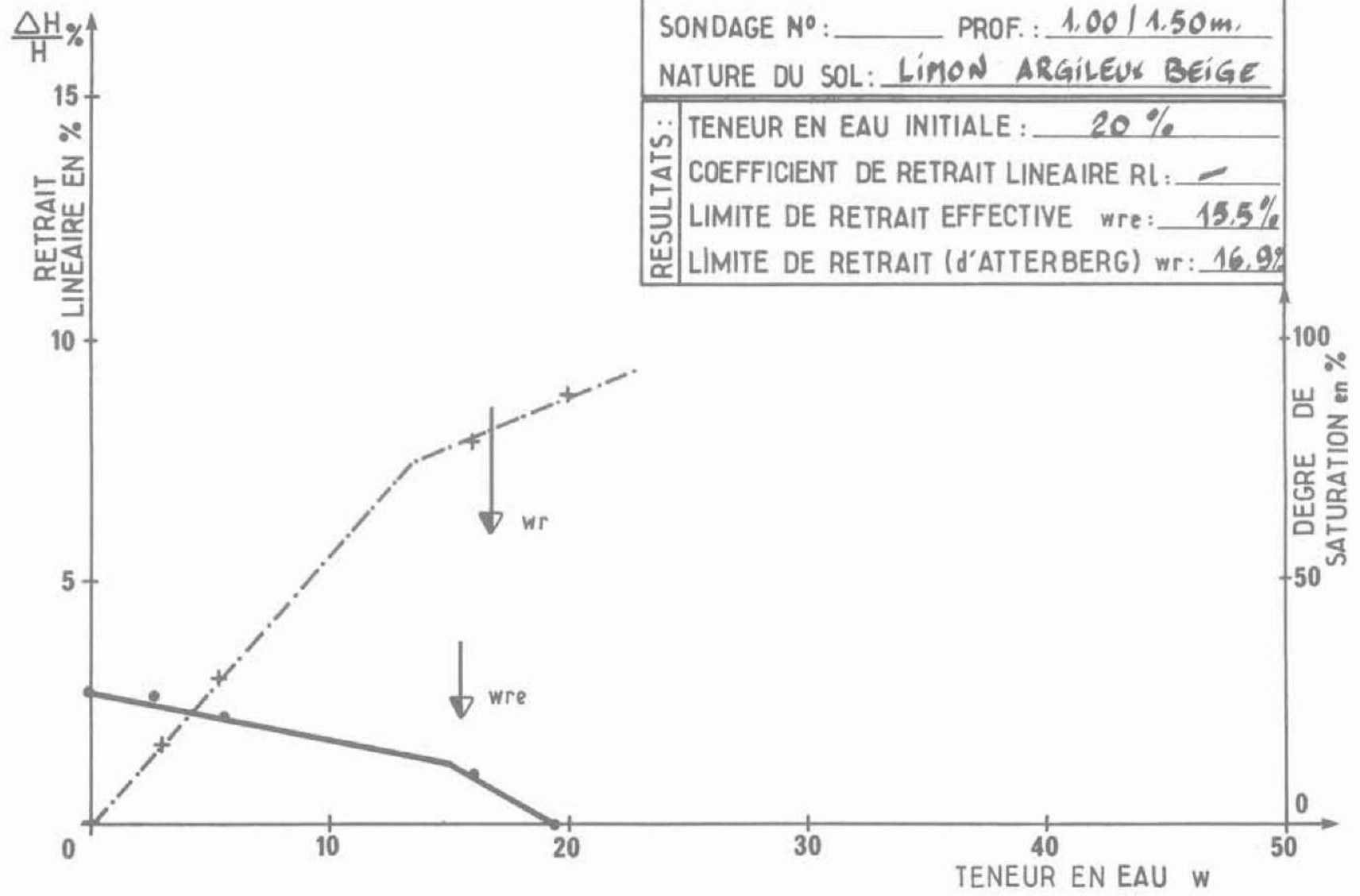

Fig. 4.1. - Essai de retrait au mercure sur du limon.

Fig. 4.1. - Shrinkage test by mercury method on silt. 
Fig. 4.2. - Essai de retrait au mercure sur du limon argileux.

Fig. 4.2. - Shrinkage tast by mercury mathod on clayed silt.

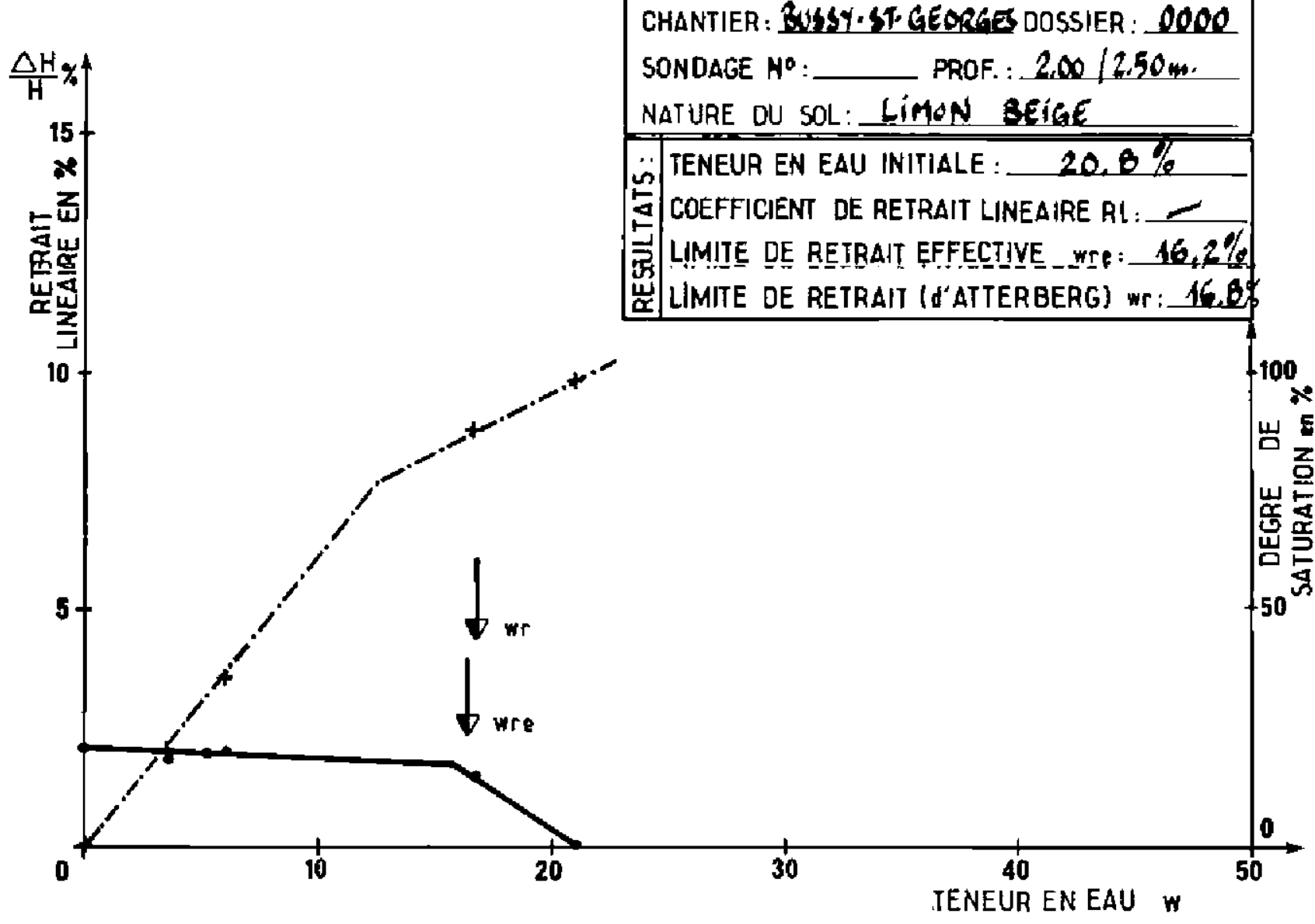

Fig. 4.3. - Essai de ratrait au mercure sur de l'argile limoneuse.

Fig. 4.3. - Shrinkage test by mercury method on silty clay.

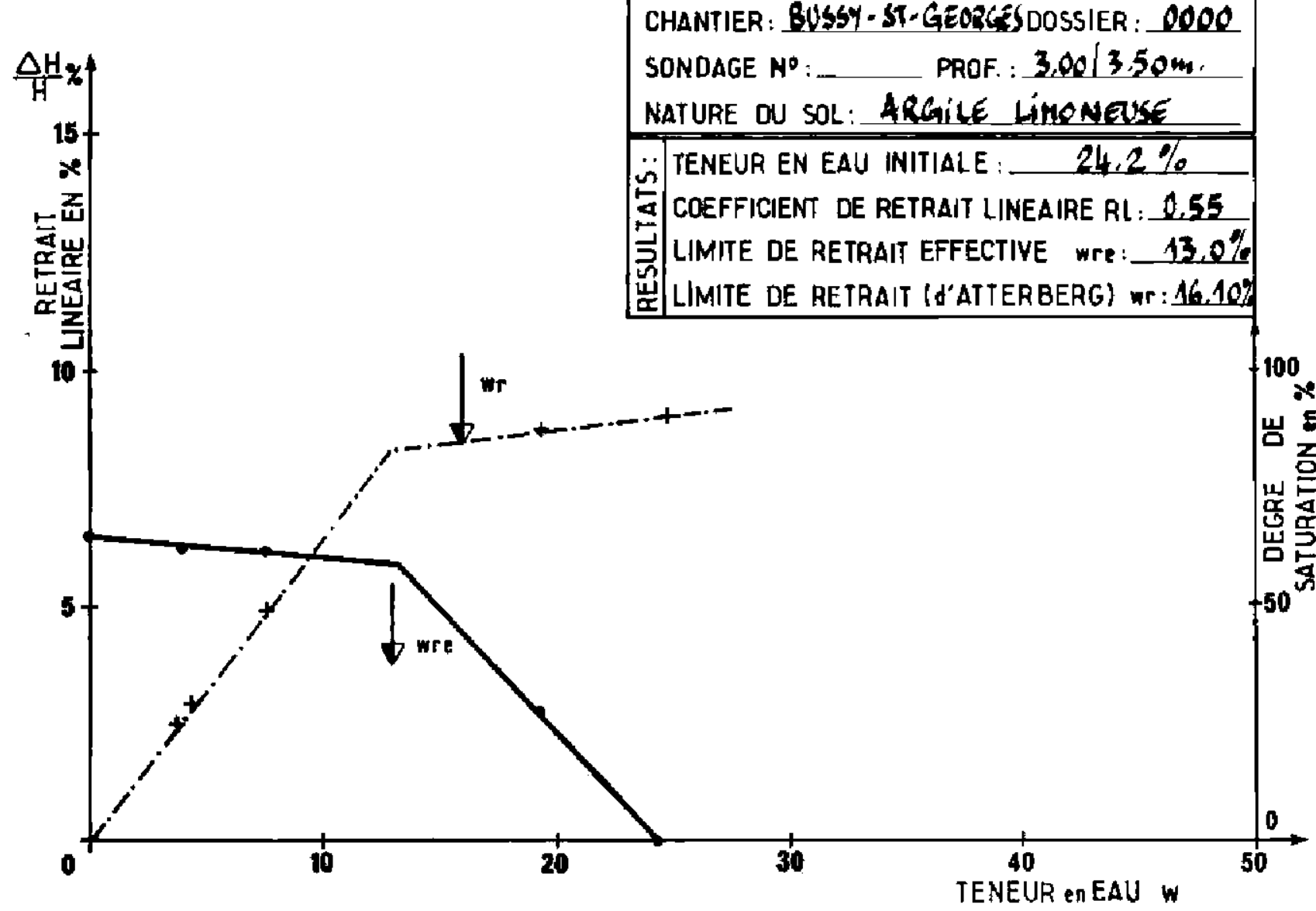




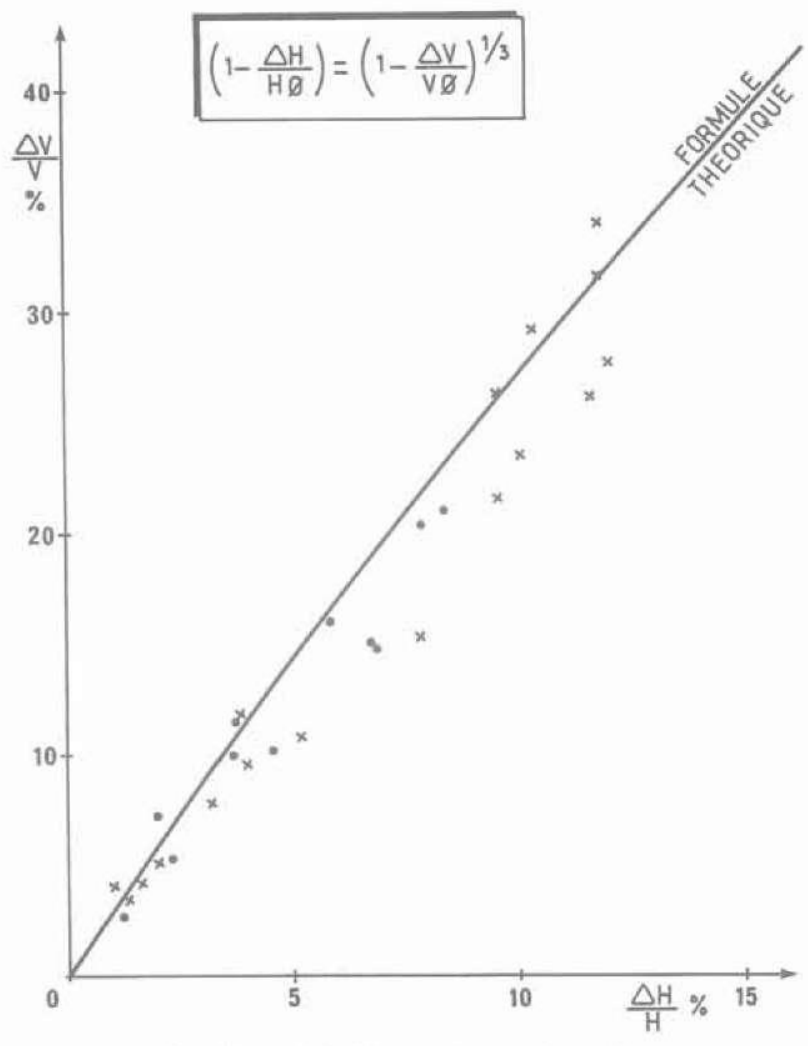

Fig. 5. - Variations dimensionnelles. Relation entre $\frac{\Delta H}{H}$ et $\frac{\Delta V}{V}$

Fig. 5. - Dimensional changes. Relation between $\frac{\Delta H}{H}$ and $\frac{\Delta V}{V}$

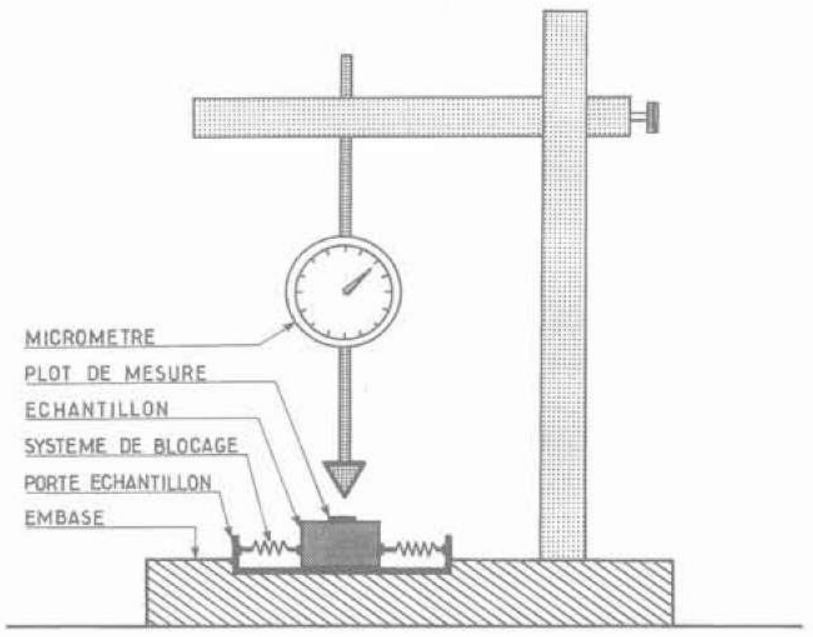

Fig. 6. - Principe d'un essai de retrait linéaire. Fig. 6. - Principle of a linear shinkage test.

\section{PRÉVISION DES MOUVEMENTS DE RETRAIT ET GONFLEMENT}

\subsection{Préambule}

Le géotechnicien n'a pas de boule de cristal pour deviner les variations futures de teneur en eau du sol sur un site donné ; à défaut, il a parfois du bon sens.
La méthodologie qui suit est basée sur le principe suivant :

- au moment de l'étude d'un cas, le sol possède un profil hydrique donné $\mathrm{PH}_{0}$ et relativement facile à mesurer ;

- si l'on estime que les conditions d'évapotranspiration ou de modification de régime de la nappe sont telles que l'on peut atteindre un nouveau profil $\mathrm{PH}_{1}$, il est possible de prévoir l'amplitude des mouvements correspondant au passage de $\mathrm{PH}_{0}$ à $\mathrm{PH}_{1}$ en supposant que l'état des contraintes totales est constant.

Si le passage de $\mathrm{PH}_{0}$ à $\mathrm{PH}_{1}$ correspondant à une réduction de teneur en eau, le phénomène sera un retrait, et dans le cas contraire un gonflement.

Notons en particulier qu'à partir des essais présentés dans la première partie, il est possible de prévoir l'amplitude des mouvements maximaux qui peuvent se produire.

Ces mouvements maximaux correspondent :

- à une chute totale de la succion du sol dans le cas du gonflement par mise en contact avec une arrivée d'eau libre;

- à une diminution de la teneur en eau telle que la limite effective de retrait soit atteinte.

Compte tenu de l'incertitude sur les variations réelles qui peuvent se produire pendant la durée de vie de l'ouvrage, la connaissance de l'amplitude des retraits et gonflements maximaux présente un intérêt tout particulier.

Notons que l'estimation des tassements à partir des essais œedométriques, en supposant classiquement l'échantillon saturé et en contact avec de l'eau libre, relève de la même démarche.

\subsection{Prévision des gonflements}

\subsubsection{Amplitude maximale de gonflement} sous un dallage (fig. 7).

Il s'agit du cas le plus simple.

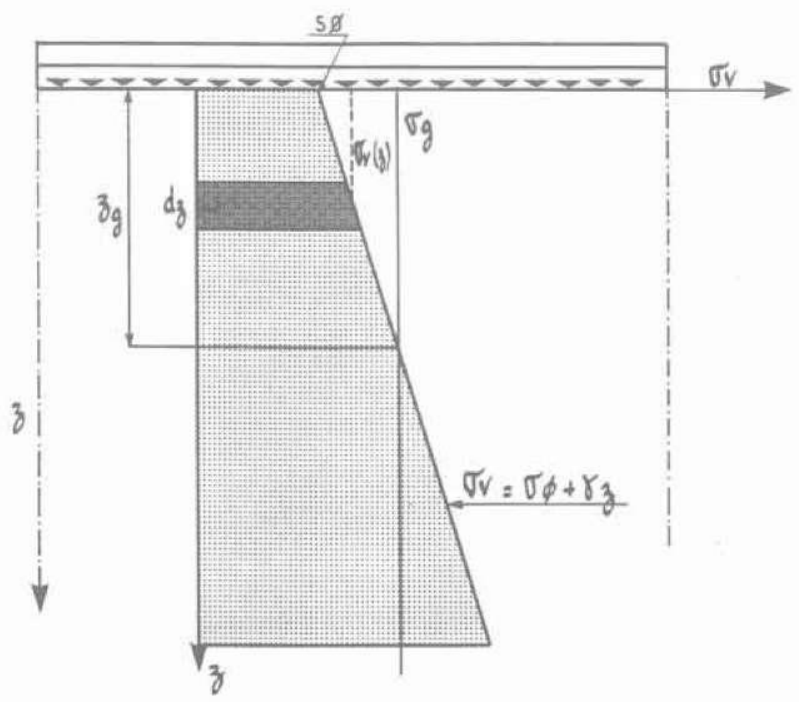

Fig. 7. - Distribution des contraintes sous un dallage uniformément chargé.

Fig. 7. - Stress distribution under a slab with uniform loading. 
On suppose ici le dallage soumis à une charge (poids propre, fondation) et surcharge constante $\mathrm{S}_{0}$.

Remarquons que dans le cas du gonflement, $\mathrm{S}_{0}$ doit être la surcharge la plus faible dont la pérennité est assurée et non la surcharge nominale, soit en pratique : $\mathrm{S}_{0}=0$.

La figure 7 présente la distribution des contraintes en profondeur sous un dallage reposant sur une couche d'argile gonflante supposée ici homogène pour la simplicité du raisonnement.

$\mathrm{z}_{\mathrm{g}}$ définit la profondeur de la zone susceptible de gonfler.

Sur cette épaisseur, l'amplitude de gonflement d'une tranche $\mathrm{dz}$ soumise à une contrainte verticale $\sigma_{\mathrm{v}}(\mathrm{z})$ est donnée selon le cas par les formules (1) ou (1 bis) :

a. Hypothèse de la linéarité :

$$
\delta H=-R G \cdot \log \left[\frac{\sigma_{g}}{\sigma_{v}(z)}\right] \cdot d z
$$

Dans le cas d'un sol homogène ( $R G=$ constante), le soulèvement final maximal en cas de saturation est :

$$
H_{g}=-R G \cdot \int_{0}^{z g} \log \left[\sigma_{g} / \sigma(z)\right] \cdot d z
$$

Pour un sol hétérogène il est aisé de faire une intégration par tranches.

\section{b. Loi non linéaire}

On procède de la même manière que précédemment avec une intégration par tranches; le gonflement de chaque tranche étant déduit de la formule (1 bis) dans le cas d'une loi hyperbolique ou directement des résultats de l'essai de gonflement.

\subsubsection{Estimation du gonflement maximal sous une semelle}

Nous supposons, toujours par simplicité de l'exposé, le sol homogène. La méthode peut être étendue facilement aux sols hétérogènes.

La figure 8 représente la distribution des contraintes sous une semelle continue selon la théorie de BOUSSINESQ.

La courbe I en trait plein représente la distribution dans l'axe.

La courbe II en pointillé, la distribution à la verticale du bord.

La courbe en trait mixte, la distribution à l'extérieur à une distance du bord de la semelle égale à 0,5 fois la largeur.

Si le sol est mis au contact d'eau libre, toutes choses égales par ailleurs, il va gonfler dans toutes les tranches où les contraintes sont inférieures à $\sigma_{\mathrm{g}}$, entraînant la semelle.
Directement sous la semelle, les contraintes ne sont pas uniformes comme nous l'avons supposé, mais il semble malgré tout optimiste de considérer que le soulèvement est dû à la seule zone 1 hachurée horizontalement,

Une estimation plus réaliste semble pouvoir être retenue en considérant la zone comprise entre $\sigma_{\mathrm{g}}$ et la courbe en pointillé (zones $1+2$ ) ou une répartition intermédiaire entre les courbes I et II.

Le soulèvement total est calculé à l'aide des formules 1 ou 1 bis avec une intégration par tranche.

\subsection{Prévision des amplitudes de retrait}

A partir des essais décrits précédemment, il est aisé de déterminer l'amplitude de l'affaissement du sol corrélatif au passage d'un état de teneur en eau $w_{1}(z)$ à un état de teneur en eau $w_{2}(z)$ avec $w_{2}<w_{1}$, quel que soit $z$.

Si $w_{f}=\max \left(w_{2}, w_{\text {re }}\right)$ avec wre = limite de retrait effective, on aura en effet:

$$
\delta \mathrm{H}=\int_{0}^{z \max }-\mathrm{R} 1\left[\mathrm{w}_{1}(\mathrm{z})-\mathrm{w}_{\mathrm{f}}(\mathrm{z})\right] \cdot \mathrm{dz}
$$

$\mathrm{R}_{1}=$ coefficient de retrait linéaire (voir § 1.3.1).

\section{PRISE EN COMPTE DES EFFORTS PARASITES SUR LES FONDATIONS PROFONDES ET SEMI-PROFONDES}

\subsection{Notion de point neutre}

On appelle point neutre, la profondeur $\mathrm{H}_{\mathrm{N}}$ du point situé dans l'axe de la fondation au-delà duquel les sols ne sont plus soumis à retrait, ni gonflement.

La détermination de ce point ne peut que relever d'une étude détaillée du site et d'observations liées au comportement des sols gonflants.

Dans le cas d'un assèchement général d'un site par suite, par exemple, de la disparition d'une nappe, il peut correspondre à la base de la couche d'argile gonflante. Dans le cas de phénomènes saisonniers, on sait que l'épaisseur affectée en terrain vierge et horizontal est sous notre climat de l'ordre de 1,5 m mais peut atteindre plusieurs mètres à proximité des certains arbres (BIDDLE, 1983) ou par exemple dans les terrains en pente lorsque ces phénomènes entraînent le développement de la solifluxion.

\subsection{Prise en compte de l'action directe} du gonflement sur la fondation elle-même (fig. 9)

La valeur maximale $F_{g}$ de la force de soulèvement due au gonflement de la couche supérieure est:

$$
F_{g}=H_{N} \cdot p * q_{s}
$$




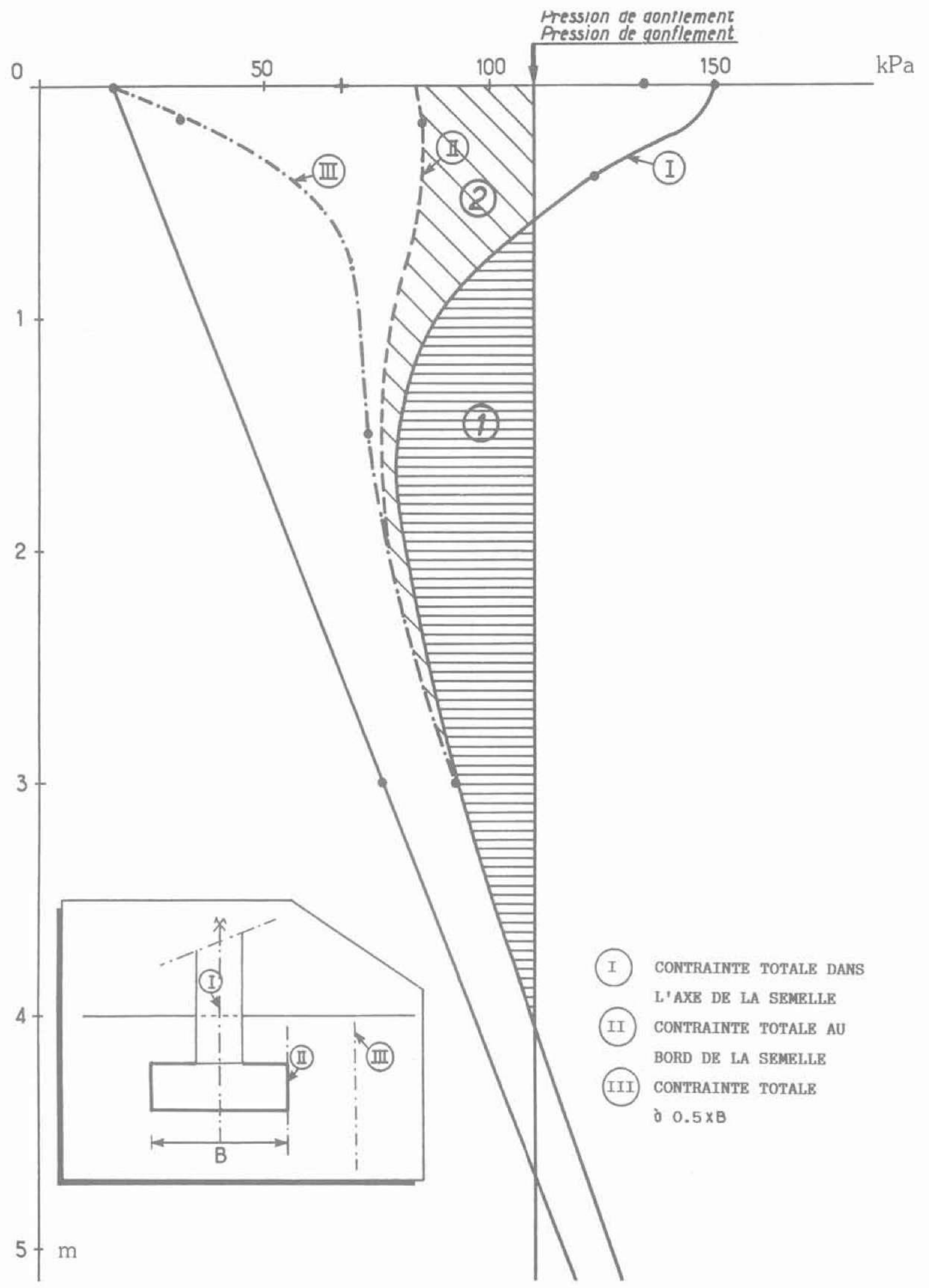

Fig. 8. - Semelle continue de $0,60 \mathrm{~m}$ de large encastrée à $0,80 \mathrm{~m}$. Répartition des contraintes dans l'axe, au bord et à l'extérieur.

Fig. 8. - Shallow foundation with a width of $0.60 \mathrm{~m}$, imbedded of $0.80 \mathrm{~m}$. Stress distribution at the axe, border and outward of the foundation. 


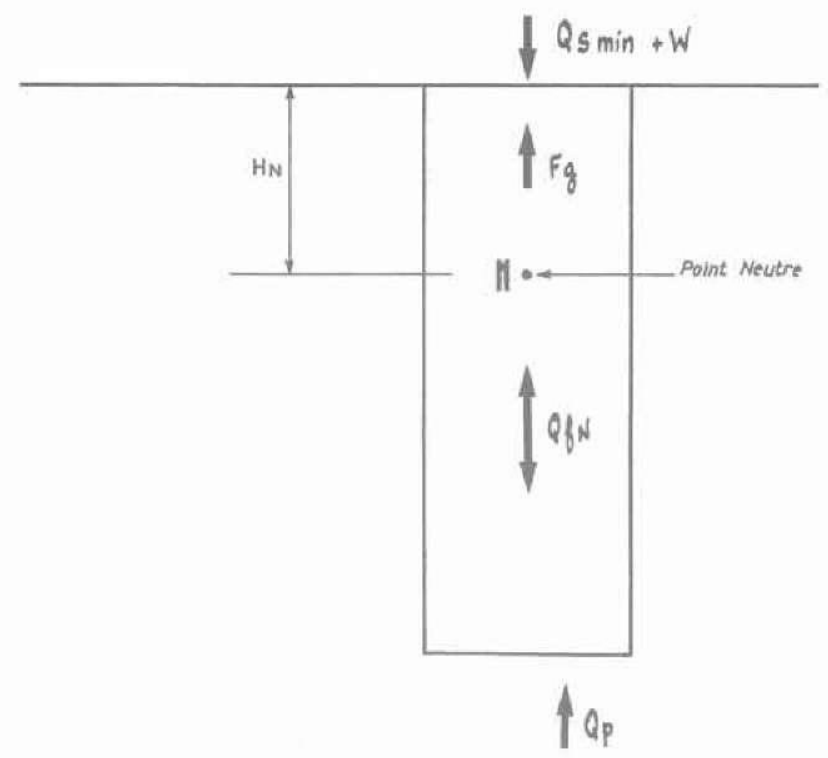

Fig. 9. - Répartition des efforts dans une fondation profonde soumise au gonflement.

Fig. 9. - Distribution of forces in a deep foundation subjected to swelling.

avec :

$\mathrm{p}=$ périmètre de la fondation ;

$\mathrm{q}_{\mathrm{s}}=$ frottement latéral unitaire limite du sol gonflant.

Appelons (fig. 9) :

$\mathrm{Q}_{\mathrm{s}} \min =$ charge de service minimale que l'on est sûr de mobiliser sur la fondation ;

$\mathrm{W}=$ poids du puits ;

$\mathrm{Q}_{\mathrm{S}} \max =$ charge maximale de service (ELS) ;

$\mathrm{Q}_{\mathrm{fN}}=$ charge mobilisable en frottement latéral sous ELS au-dessous de $\mathrm{N}$;

$\mathrm{Q}_{\mathrm{pN}}=$ charge mobilisable en pointe sous ELS.

Condition de non-soulèvement :

La condition suivante doit être vérifiée (outre les justifications habituelles) :

$$
\mathrm{W}+\mathrm{Q}_{5} \min +\mathrm{Q}_{\mathrm{fN}}>\mathrm{F}_{\mathrm{G}}
$$

Condition de résistance à la traction:

Si $Q_{5} \min -F_{g}>0$, la fondation est totalement en compression.

Dans le cas contraire, il faut vérifier que la contrainte de traction $\sigma_{t}=\left(\mathrm{F}_{\mathrm{g}}-\mathrm{Q}_{\mathrm{S}} \mathrm{min}\right) / \mathrm{S}$ est admissible pour le matériau constitutif de la fondation ou prendre des mesures nécessaires (armatures).

\section{Nota :}

On peut se poser la question de savoir s'il faut considérer le gonflement comme une sollicitation exceptionnelle et remplacer $Q_{f N}$ par $Q_{f}$ mobilisable sous ELU.

\subsection{Cas particulier des reprises en sous-œuvre} Gonflement empêché : action du gonflement sur les anciennes fondations (fig. 10 et 11)

Lorsque d'anciennes fondations superficielles reposant sur une argile gonflante est reprise en sous-œuvre, il faut:

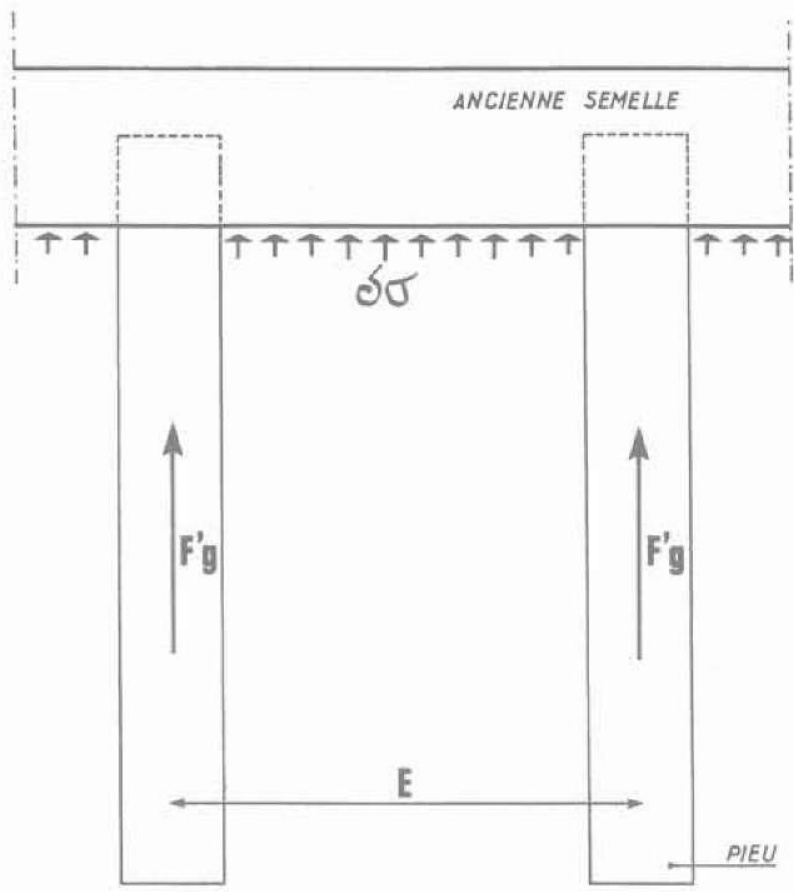

Fig. 10. - Action de gonflement sous les anciennes semelles. Fig. 10. - Action of swelling under ancient shallow foundations.

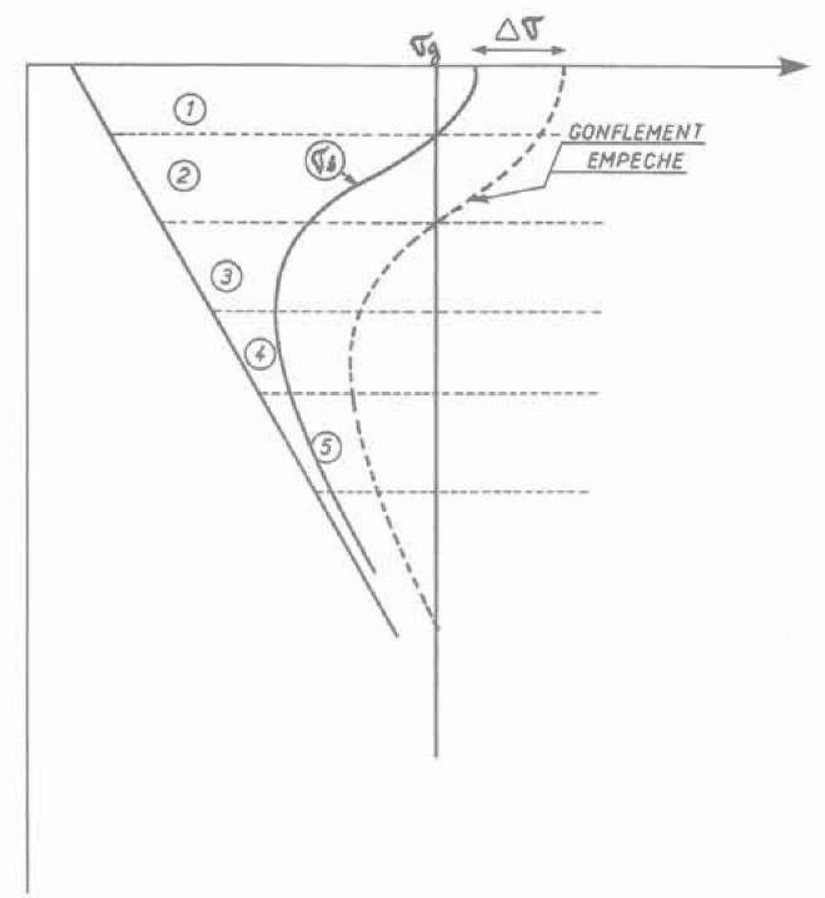

Fig. 11. - Comportement d'une semelle sous gonflement empêché.

Fig. 11. - Behaviour of a foundation with impered swelling.

- soit désolidariser la fondation ancienne du sol d'assise (mais cela n'est généralement pas possible, ni recommandable) ;

- soit rajouter à $F_{g}$, la poussée $F_{g}$ résultant de la contrainte de soulèvement $\delta \sigma$ qui peut s'exercer sur la sous-face de lancienne semelle (fig. 10).

Sur la figure $11, \sigma_{\mathrm{g}}$ est la pression de gonflement. 
$\sigma_{\mathrm{s}}$ est la contrainte de service sous la semelle.

$\delta_{\sigma}$ est l'augmentation moyenne des contraintes dues au gonflement.

Si on suppose la semelle bloquée par les pieux, la valeur de $\delta \sigma$ s'établit de façon à ce que la compressibilité supplémentaire dans les tranches telles que 1 et 2 où la contrainte finale est supérieure à $\sigma_{\mathrm{g}}$ équilibre le gonflement des tranches telles que 3 à 5 où la contrainte finale est inférieure à $\delta_{\mathrm{g}}$.

On constate sur ce graphique qu'en cas de nonsoulèvement de la semelle, la pression exercée sur celle-ci peut être supérieure à la pression de gonflement. Dans des cas extrêmes, elle peut théoriquement atteindre la pression limite du sol (écoulement du sol de part et d'autre de la semelle).

Il est relativement aisé de pratiquer une intégration par tranches. Les tassements des tranches comprimées sont calculés classiquement, par exemple à partir du module œedométrique et les gonflements des tranches gonflantes sont calculés par les formules 1 ou 1 bis.

On procède par approximations successives pour définir la contrainte sous la semelle conduisant à une déformation globale nulle (équilibre des tassements et gonflements).

La difficulté réside dans le fait que l'on ne peut se contenter de raisonner dans laxe de la semelle, comme nous l'avons vu précédemment.

\subsection{Remarques diverses}

a. Certains auteurs préconisent de chemiser les puits sur la hauteur sensible. Si le sol sous le niveau d'assise des reprises en sous-ceuvre est toujours gonflant, nous estimons au contraire qu'il faut couler à pleine fouille tout au moins sous nos climats pour éviter de créer un chemin préférentiel d'infiltration des eaux vers le niveau d'assise de la fondation.

Par contre, si le sol d'assise est insensible à l'eau, il est effectivement intéressant d'éliminer le frottement latéral.

b. En ce qui concerne les fondations semi-profondes, on peut utiliser la méthode de calcul préconisée dans l'annexe 2 ci-jointe, en intégrant l'effort parasite $F_{G}$ dû au gonflement.

\subsection{Prise en compte du retrait}

L'opération est plus facile car le retrait au droit des anciennes fondations (reprise en sous-œuvre) conduit à un décollement et n'entraîne pas d'efforts parasites.

L'action directe sur la fondation conduit à un effort parasite $F_{R}$ selon un processus identique à celui du frottement négatif.

Comme précédemment, on peut en première approximation admettre que la valeur maximale de $F_{r}$ peut atteindre :

$$
F_{\mathrm{r}}=\mathrm{H}_{\mathrm{N}} \cdot \mathrm{q}_{\mathrm{s}} \cdot \mathrm{p}
$$

Cette charge $F_{r}$ doit être ajoutée aux autres sollicitations de la fondation.

Bien entendu, la résistance de la fondation est calculée en ne considérant que le frottement positif au-delà de $\mathrm{H}_{\mathrm{N}}$,

\subsection{Reprises en sous-œuvre. Autres efforts parasites}

Lorsque une reprise en sous-œuvre est réalisée, les nouvelles fondations ne sont pas toujours centrées sur les anciennes. De plus dans le cas de pieux, ceux-ci sont parfois inclinés pour des nécessités matérielles de réalisation.

Notamment lorsque les reprises en sous-œuvre sont faites avec des micropieux de faible inertie, il est nécessaire de justifier les fondations en prenant en compte les efforts parasites dus à l'excentrement et l'inclinaison, lorsqu'il n'est pas possible de les combattre en entrecroisant les pieux.

\section{a. Excentrement}

$V$ étant l'effort vertical à reprendre, l'excentrement conduit à un moment en tête $\mathrm{M}=\mathrm{V}$.e.

\section{b. Inclinaison}

L'inclinaison $\beta$ conduit à un effort transversal :

$$
\mathrm{T}=\mathrm{V} \cdot \sin \beta \text {. }
$$

La répartition des moments dans le micropieu doit être calculée, par exemple par une méthode élastoplastique, et le micropieu conçu en conséquence.

Remarquons à cet effet que la présence d'un massif ou de longrines enterrées à forte inertie (Is), dans lesquels le micropieu à faible inertie (Ip) est encastré, réduit considérablement les efforts parasites dans le micropieu, même s'il est enterré dans des terrains médiocres. Le massif doit être pris en compte dans les calculs (fig. 12).

\section{BIBLIOGRAPHIE}

PELTIER R. (1959), Manuel du Laboratoire Routier, Dunod.

PHILIPPONNAT G. (1978), Désordres dus à la présence des sols gonflants dans la région parisienne. Annales ITBTP, $n^{\circ} 364$.

DRISCOLL R. (1983), The influence of vegetation on swelling and shrinkage of clays soils in Britain. Géotechnique, vol. 33, $\mathrm{n}^{\circ} 2$.

BIDDLE P.G. (1983), Patterns of soil drying and moisture deficit in the vicinity of trees on clay soils. Géotechnique, vol. $33, \mathrm{n}^{\circ} 2$.

ASTM (1985), Standard test methods for onedimensional swell or settlement potential of cohesive soil.

PHILIPPONNAT G. (1987), Sols expansifs en France. Identification et recommandations pour les fondations. 6th International Conference on Expansive Soils, New Delhi, India. 


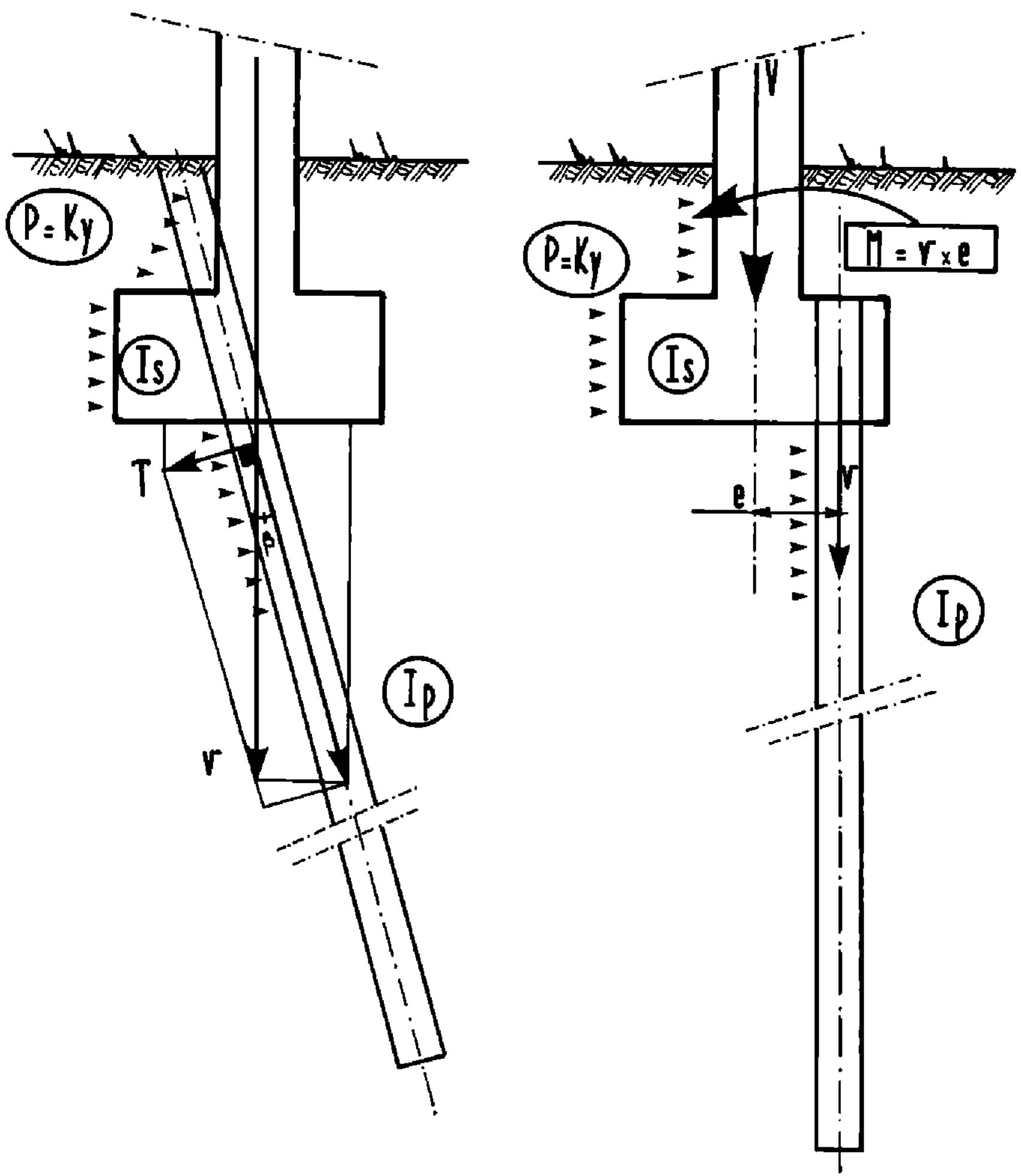

Fig. 12. - Efforts parasites sur une reprise on sous-ceuvre.

Fig. 12 - Secondary forces on undarpinning.

DIDIER G. et al. (1987). Etude à l'cedomètre du gon. flement des sols. IX European conference of soils mechanics and foundation engineering

MOUROUX P. et al. (1988), La construction économique sur sols gonfiants, BRGM Manuels et Méthodes $n^{\circ} 14$
CHEN F.H. (1988), Foundation on expansive soils. Elsevier.

TECHNICAL COMMITTEE OF ISSMFE (TC6) (1990). Draft standard evaluation of swelling pressure of expansive soils in laboratory. Central Board of Irrigation and Power, Malcha Marg. Chanakyapuri, New Delhi, India. 


\title{
ANNEXE 1 \\ Avant-projet \\ Evaluation de la Pression de Gonflement de Sols Gonflants en Laboratoire (traduction)
}

\author{
Comité Technique sur les Sols Expansifs \\ (TC6) de l'ISSMFE
}

Président :

Prof. R.K. Katti

Director and Consultant,

Universal Earth Engineering Consultancy

Services Pvt. Ltd.

401-B, Poonam Chambers

Shivsagar Estate, Dr. A.B. Road

Worly, Bombay-40018, India.

\section{Secrétaire :}

Mr. K.R. Saxena

Director

Central Board of Irrigation and Power

Malcha Marg, Chanakyapuri

New Delhi-110021, India.

\section{Membres :}

Prof. Silvano J. Trevisan, Argentina.

Dr. P.W. Mitchell, Australia.

Dr. Balu Iyer, Canada.

Mr. Xi-Ling Huang, China.

Mr. M. Londez, France.

Mr. G. Philipponnat, France.
Mr. Aris C. Stamatopoulos, Greece.

Dr. L. Rethati, Hungary.

Dr. N.V. Nayak, India.

Dr. C. Behnia, Iran.

Prof. Joseph G. Zeithlen, Israel.

Prof. Ing. Ginseppe Scarpelli, Italy.

Prof. S.A. Ola, Nigeria.

Prof. G.E. Blinght, South Africa.

Dr. A.A.B. Williams, South Africa.

Dr. V. Escario, Spain.

Prof. J.A. Jimenez Salas, Spain.

Mr. Richard Driscoll, UK.

Dr. Robert L. Lytton, USA.

Prof. E.A. Sorochyan, USSR.

Mr. M.F.C. Warren, Zimbabwe.

Nominations attendues de :

Morocco

Pakistan

Southeast Asia

Switzerland

Syria

Central board of irrigation and power

Malcha Marg, Chanakyapuri New Delhi, INDIA

\section{OBJECTIF}

En génie civil, les caractéristiques de gonflement des sols expansifs, in situ ou remaniés lorsqu'ils sont utilisés comme matériaux de construction, doivent être traités séparément.

Actuellement, il n'existe pas de consensus sur la définition du potentiel de gonflement (aussi bien variation de volume que pression), bien que des métho- des expérimentales quantitatives de terrain et de laboratoire aient été adoptées par plusieurs pays.

Les spécifications ci-dessous donnent le détail de la méthode pour définir la pression de gonflement en utilisant la méthode de l'œdomètre.

\section{TERMINOLOGIE}

Les définitions suivantes sont utilisées : 


\subsection{Pression de gonflement}

C'est la pression que le sol expansif exerce lorsque son gonflement est empêché.

La notion de la pression de gonflement est purement expérimentale et peut ne pas avoir la même signification que celle des forces répulsives entre les molécules, nommée pression de gonflement par les physiciens.

\subsection{Chargement}

Le chargement fait référence à une contrainte totale et non à une contrainte effective.

\section{APPAREILLAGE}

\subsection{CEdomètre}

Un appareil permettant de maintenir l'échantillon dans une bague fixe ou mobile avec des pierres poreuses (ou disques en céramique) sur chaque face de l'échantillon.

Un œdomètre doit permettre en outre la possibilité de submerger l'échantillon, d'appliquer une charge verticale et de mesurer la variation de l'épaisseur de l'échantillon et sa pression de gonflement.

Le système de fixation du capteur doit être rigide et permettre une mesure précise de l'expansion verticale de l'échantillon.

\subsubsection{Diamètre et épaisseur de l'échantillon}

L'échantillon a un diamètre de $75 \mathrm{~mm}$ et une épaisseur de $20 \mathrm{~mm}$.

\subsubsection{Bague}

La bague doit être faite d'un matériau non corrosif et doit avoir un côté tranchant de façon à ce que l'échantillon soit introduit avec le moins de remaniement possible.

La bague doit être placée de manière à ce que la partie tranchante soit dirigée vers le haut et logée de façon à ne pas endommager cette partie. Elle doit être munie d'une rehausse de même diamètre et de hauteur effective de $20 \mathrm{~mm}$. Cette rehausse repose sur la bague de l'échantillon.

\subsubsection{Pierres poreuses}

Ces pierres doivent avoir une grande perméabilité en comparaison de celle de l'échantillon de sol.

Le diamètre de la pierre poreuse supérieure doit être de 0,2 à $0,5 \mathrm{~mm}$ inférieur au diamètre intérieur de la bague. Son épaisseur est au minimum de $15 \mathrm{~mm}$.

La pierre poreuse supérieure doit être maintenue par une plaque perforée rigide d'au moins $10 \mathrm{~mm}$ d'épaisseur, pour éviter la rupture de la pierre et pour permettre le libre drainage de l'eau.

\subsubsection{Réservoir d'eau}

Une burette de volume suffisant pour alimenter l'échantillon en eau.

\subsubsection{Autres équipements de laboratoire}

Des outils pour la découpe de l'échantillon, scie à fil fin, couteau, spatule, etc. pour tailler l'échantillon au diamètre intérieur de la bague de l'œdomètre avec le minimum de remaniement, une étuve, un dessiccateur, des balances, etc.

\section{PRÉPARATION DE L'ÉCHANTILLON D'ESSAI}

\subsection{Préparation de l'échantillon à partir de sol remanié}

Dans le cas de sol remanié, celui-ci doit être compacté à la densité et à la teneur en eau désirée (in situ) dans un moule de compactage standard Proctor, l'échantillon est alors prélevé en enfonçant la bague de l'œdomètre. L'échantillon doit ensuite être lissé et arasé aux niveaux supérieur et inférieur de la bague et les vides éventuels doivent être comblés. La bague comprenant l'échantillon est pesée. La perte d'eau de l'échantillon doit être la plus faible possible pendant la préparation.

\section{Notes}

a. Dans le cas d'échantillons de sol remanié, la teneur en eau initiale doit être égale à la limite de retrait ou à la teneur en eau in situ, durant la saison sèche, ainsi la pression de gonflement obtenue sera maximale.

b. L'essai Proctor se fait sur des échantillons séchés à l'air dans le cas où le séchage à l'étude peut influer sur le comportement.

c. Si les conditions de mise en place in situ sont inférieures à la teneur en eau de l'optimum Proctor, l'essai Proctor est nécessaire pour des échantillons séchés à l'air.

d. Des corrections de densité et de teneur en eau sont effectuées si des restrictions doivent être imposées sur la dimension maximale des grains (une dimension max. des grains de $1 / 10$ de $20 \mathrm{~mm}$ est convenable).

\subsection{Préparation à l'échantillon à partir de sol non remanié}

Notes

a. La procédure recommandée par TC24 de l'ISSMFE sera adoptée.

b. La teneur en eau in situ la plus faible enregistrée sur une longueur période est préférable.

\section{PROCÉDURE DE L'ESSAI}

\subsection{Montage}

5.1.1. La bague contenant l'échantillon doit être placée entre deux pierres poreuses désaérées avec un papier filtre entre l'échantillon de sol et les pierres poreuses. Le piston de chargement doit alors être centré sur le dessus de la pierre poreuse. 
5.1.2. Ce montage doit être placé sur le bâti de chargement. La charge est appliquée sur l'échantillon sans excentrement. Un système direct de mesure de déformation est adapté sur la cellule. L'échantillon est inondé d'eau distillée en permettant son gonflement sous une charge spécifique.

\subsection{Chargement}

L'essai doit être conduit pour au moins trois chargements (couvrant une gamme allant du gonflement libre à $100 \mathrm{kPa}$, mais pour des conditions extrêmes la charge la plus élevée peut varier selon les conditions spécifiques et une autre séquence de chargement peut être adoptée) en saturant l'échantillon jusqu'à ce que l'équilibre soit atteint.

\subsection{Résultats d'essai}

Pour chaque charge, les lectures du comparateur doivent être relevées jusqu'à ce que l'équilibre soit atteint. Ceci est vérifié en traçant les points de mesures de gonflement en fonction du temps sur un graphe semilog. Le point devenant asymptotique avec l'abscisse (échelle de temps) donne la pression de gonflement à l'équilibre.

\section{PRÉSENTATION DES RÉSULTATS}

Des études sur les variations de volume de plusieurs sols remaniés et non remaniés à l'œedomètre ont montré qu'il existait une relation linéaire entre le pourcentage de gonflement et le logarithme naturel de la charge appliquée.

Des résultats typiques d'essai sont indiqués sur la figure A1.

Le pourcentage de gonflement sous une charge quelconque peut par conséquent être estimé après avoir déterminé le gonflement libre et la pression de gonflement du sol.

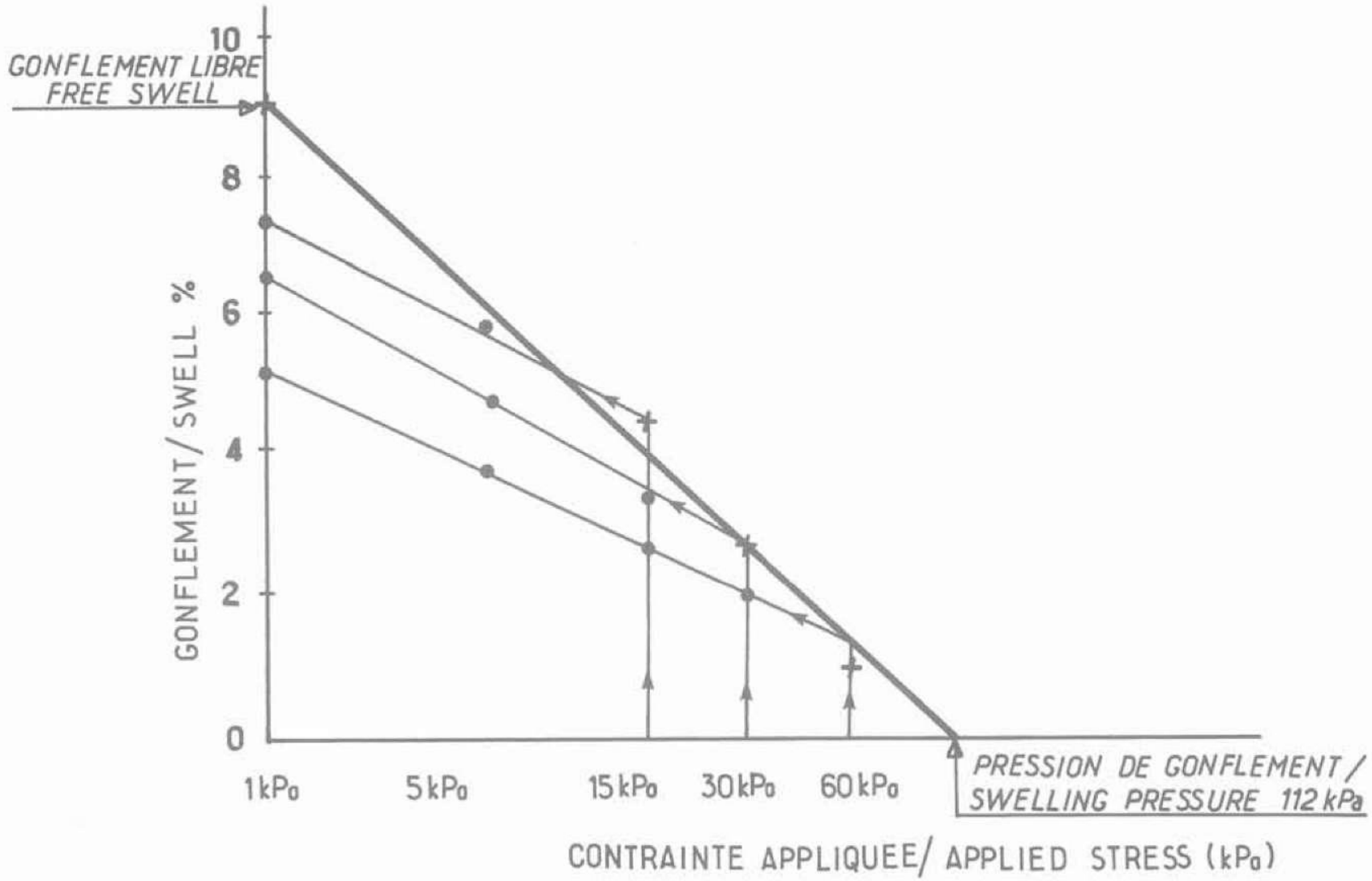

Fig. A1. - Pourcentage de gonflement/Pression appliquée.

Fig. A1. - Swell percentage vs applied stress. 


\section{ANNEXE 2 \\ Fondations semi-profondes: méthode de calcul}

\section{Références :}

- Méthode pressiométrique - DTU 13-12 de mars 1988 ;

- Fondations et ouvrages en terre - Gérard PHILIPPONNAT, Eyrolles 1979 - Chapitre VII « Méthode de calcul des fondations semi-profondes ";

- Calcul d'une fondation mixte semelle-pieux sous charge verticale centrée. Note d'information technique - O. COMBARIEUX, LCPC, 1988 .

- Estimation par les paramètres pressiométriques de l'enfoncement sous charges axiales de pieux forés dans les sols fins - FRANK et ZHAO, Bulletin de liaison des LPC $\mathrm{n}^{\circ} 119$ (1982).

\section{MÉTHODE DE CALCUL}

\subsection{Détermination de la contrainte ultime qu}

\subsubsection{Contrainte ultime $q_{p u}$ due à la résistance} à la base du puits

La contrainte ultime est donnée par la formule:

$$
\mathrm{q}_{\mathrm{pu}}=\mathrm{k}_{\mathrm{p}} \cdot \mathrm{p}_{\mathrm{le}} e^{\circ}+\gamma \mathrm{D}
$$

avec :

$\mathrm{P}_{\mathrm{le}} \cdot$ = pression limite nette équivalente de la couche d'ancrage ;

$k_{\mathrm{p}}=$ facteur de portance fonction de D/B, D étant pris égal à l'encastrement équivalent selon $\mathrm{L}$. MENARD

$\gamma \mathrm{D}=$ poids des terres sur la hauteur minimale d'encastrement de la fondation.

\subsubsection{Calcul du frottement latéral limite $Q_{f l}$}

Le frottement latéral Qf ne peut être pris en compte que si le puits est coulé à pleine fouille.

En l'absence de frottements parasites (frottement négatif, actions du retrait ou du gonflement, ...), il est calculé en négligeant le frottement sur une hauteur du fût du puits de $1 \mathrm{~m}$ à partir de la surface du sol, les terrains de surface étant généralement remaniés et déconsolidés lors des travaux:

$$
\mathrm{Q}_{\mathrm{fl}}=\mathrm{P}_{\mathrm{e}} \cdot \Sigma \mathrm{q}_{\mathrm{si}} \cdot \mathrm{h}_{\mathrm{i}}
$$

avec :

$\mathrm{p}_{e}=$ périmètre du puits ;

$\mathrm{q}_{\mathrm{si}}=$ frottement latéral unitaire de la couche $\mathrm{i}$;

$h_{\mathrm{i}}=$ épaisseur traversée par le puits dans la couche $\mathrm{i}$.

\subsubsection{Valeur globale}

La charge limite des puits est donnée par la formule:

$$
Q_{u}=q_{p u} \cdot A+Q_{f l}
$$

avec : $\mathrm{A}=$ section droite du puits

La contrainte ultime correspondante est $\mathrm{q}_{u}=\mathrm{Q}_{\mathrm{u}} / \mathrm{A}$.

1.2. Contrainte de calcul en vue de la justification aux états limites ultimes

La contrainte de calcul est selon le DTU 13-12 :

$$
\mathrm{q}=\frac{\mathrm{q}_{\mathrm{u}}}{2}
$$

Nota :

- La vérification aux ELU vis-à-vis de q doit prendre en compte le poids propre du puits et d'éventuels frottements parasites.

- Si l'importance des tassements conduit à réduire la contrainte maximale sous ELS, la contrainte de calcul sous ELU est réduite dans les mêmes proportions.

\subsection{Estimation de la charge nominale $\mathrm{Q}_{\mathrm{N}}$} sous états limites de services

En tenant compte du poids propre du puits qui doit être inclus dans $Q_{N}$, la charge nominale sous ELS peut être estimée comme suit :

$$
\mathrm{Q}_{\mathrm{N}}=\mathrm{A} \cdot \mathrm{q}_{\mathrm{pN}}+\mathrm{Q}_{\mathrm{N}}
$$

avec :

$\mathrm{Q}_{\mathrm{N}}=$ charge nominale sous ELS

$A=$ section du puits;

$\mathrm{q}_{\mathrm{pN}}=$ résistance nominale de pointe sous ELS ;

$$
\mathrm{q}_{\mathrm{pN}}=\left(\frac{\mathrm{q}_{\mathrm{pu}}-\gamma \mathrm{D}}{3}\right)+\gamma \mathrm{D}
$$

$\mathrm{Q}_{\mathrm{fN}}=$ frottement latéral autorisé sous $\mathrm{ELS}=\mathrm{Q}_{\mathrm{fL}} / 2$. La contrainte de service autorisée sous ELS est :

$$
\mathrm{p}=\mathrm{Q}_{\mathrm{N}} / \mathrm{A}
$$

\section{Remarques importantes}

La contrainte de calcul a été déterminée avec le même coefficient de sécurité vis-à-vis de la rupture que pour les fondations superficielles.

Du fait du déplacement faible nécessaire pour mobiliser le frottement latéral, la contrainte nominale destinée à limiter les tassements est calculée en ce qui concerne ce dernier avec la même règle que pour les pieux.

Il s'ensuit que généralement, la contrainte de calcul à ne pas dépasser sous ELU est relativement peu élevée par rapport à la contrainte nominale autorisée sous ELS. Le plus souvent, ce sont les ELU qui imposeront le dimensionnement des puits. 


\subsection{Evaluation des tassements}

\subsubsection{Loi de déformations à la base}

On considère que le puits se comporte comme un solide incompressible ce qui, dans le cas présent, est tout à fait justifié, la déformation verticale est la même en chaque point du massif.

Sous la base, le tassement est donné par les formules de L. MÉNARD reprises dans le DTU 13-12 "Fondations superficielles".

Le tassement $\mathrm{s}$ de l'assise est la somme de deux termes:

- $s_{c}$ : tassement dit de consolidation :

$-s_{d}$ : tassement dit déviatorique.

Soit :

avec :

$$
s=s_{c}+s_{d}
$$

$$
\begin{gathered}
s_{c}=\frac{\alpha}{9 E_{s}}\left(q_{p}-q_{o}\right) \cdot L c \cdot B \quad(6 \text { bis }) \\
s_{d}=\frac{2}{9 E d}\left(q_{p}-q_{o}\right) \cdot B_{o}\left(L_{d} \cdot B / B_{o}\right)^{\alpha} \quad(6 \text { ter })
\end{gathered}
$$

$B_{0}=0,6 \mathrm{~m}$;

$\mathrm{B}=$ largeur ou diamètre des puits ;

$\mathrm{q}_{\mathrm{p}}=$ contrainte réelle transmise à la base du puits sous ELS ;

$\mathrm{q}_{\mathrm{o}}=$ poids initial des terres au niveau de la base du puits ;

$\alpha=$ coefficient rhéologique dépendant de la nature du sol ;

$\mathrm{L}_{c}$ et $\mathrm{L}_{\mathrm{d}}=$ coefficients de forme ;

$\mathrm{E}_{\mathrm{s}}=$ module pressiométrique sphérique sur une épaisseur de 0,5 B sous le niveau de la base du puits

$\mathrm{E}_{\mathrm{d}}=$ module pressiométrique déviatorique pondéré selon la méthode de L. MENARD.

Nota :

En fait la formule 6 ter est remplacée par une formule plus complexe (O. COMBARIEU, 1988) qui permet d'obtenir une estimation des déformations plastiques sous charges élevées sans modifier sensiblement la valeur des tassements dans le domaine élastique.

\subsubsection{Loi de déformations en latéral}

La loi de mobilisation du frottement latéral en fonction de s s'écrit (O. COMBARIEU, 1988) :

$$
\mathrm{s}=\frac{-2 \cdot \mathrm{R}_{\mathrm{p}} \cdot \mathrm{q}_{\mathrm{s}}}{\mathrm{K}^{\prime} \cdot \mathrm{E}} \cdot \operatorname{Ln}\left(1-\frac{\tau}{\mathrm{q}_{\mathrm{s}}}\right)
$$

avec :

$\mathrm{R}_{\mathrm{p}}=$ rayon moyen du puits $=\mathrm{P}_{e} / 2 \pi$, limité supérieurement à $1 \mathrm{~m}$;

$\mathrm{q}_{\mathrm{s}}=$ frottement unitaire latéral limite;

$\mathrm{E}=$ module pressiométrique ;

$K^{\prime}=$ coefficient $(0,8$ dans les sables et les graviers et 2,0 dans les autres sols) :

$\tau=$ contrainte de frottement latéral mobilisée $(\tau \leq$ qs).

Nota :

La formule (7) fournit un déplacement proportionnel au rayon de la fondation, ce qui conduit à une aberration pour les fondations de grandes dimensions.

Compte tenu de la gamme de diamètres à l'intérieur de laquelle la formule a été validée (FRANK et ZHAO, 1982) et des ordres de grandeurs des lois de comportement en frottement latéral, il est judicieux de borner supérieurement $R_{p}$ à $1 \mathrm{~m}$.

\subsubsection{Tassement global}

En définitive, pour chaque valeur donnée à s, les formules (6) et (7) permettent de calculer la charge totale $\mathrm{Q}$ correspondante appliquée sur le puits :

$$
Q=Q_{p}+Q_{f}
$$

avec :

$$
\begin{gathered}
Q_{p}=A \cdot q_{p} \\
Q_{f}=p_{e} \cdot \Sigma h_{i} \cdot \tau_{1}
\end{gathered}
$$

Le calcul peut être exécuté par ordinateur à l'aide du programme "PUITS " de la Société SOPENA. 